\title{
Frailty and Perioperative Outcomes
}

\author{
Ranjit Deshpande ${ }^{1} \cdot$ William Stuart Asch ${ }^{1} \cdot$ Maricar Malinis $^{1}$ \\ Published online: 11 May 2018 \\ (C) Springer Science+Business Media, LLC, part of Springer Nature 2018
}

\begin{abstract}
Purpose of Review Frailty is associated with serious adverse outcomes, such as disability, health care utilization, and death in the perioperative period. In this review, we discuss surgical outcomes in a frail individual, and risk stratification for a frail individual. Recent Findings Frailty is associated with increased length of hospital stay and 30- and 90-day mortality. The data on association of frailty on discharge to home and quality of life is scant, but it is likely that they are closely related. Prehabilitation involving a diet and exercise program seems to improve outcomes in the frail surgical candidates.

Summary Making a surgical decision in frail patients is complex. Frailty is commonly associated with the older age group. The balance between high operative risk and the potential benefits of surgery is not always clear. The challenge lies in effectively identifying patients that will benefit most from operative intervention and then optimize them for surgery.
\end{abstract}

Keywords Frailty $\cdot$ Perioperative outcomes $\cdot$ Perioperative mortality $\cdot$ Frailty trajectories $\cdot$ Frailty measures $\cdot$ Frailty improved outcomes $\cdot$ Prehabilitation

\section{Introduction}

Frailty is recognized as a clinical syndrome and is comprised of a constellation of features including reduced physical activity and energy, weight loss, weakness, and slowed walking speed. Initially pioneered in the field of geriatrics, the concept of frailty applies broadly across many areas and subspecialties in medicine. In the recent years, there has been an increasing focus on frailty and its interactions with disease states and recovery. Frailty has been noted to predict adverse outcomes, such as disability, health care utilization, and death. Fifteen percent of non-nursing home population 65 years or older are frail; out of this population, $42 \%$ were hospitalized in the previous years [1]. Frail patients are vulnerable and predicted

This article is part of the Topical Collection on Geriatric Anesthesia

Ranjit Deshpande

Ranjit.deshpande@yale.edu

William Stuart Asch

William.asch@yale.edu

Maricar Malinis

Maricar.malinis@yale.edu

Yale University School of Medicine, New Haven, CT, USA to have a decreased reserve leading to an inability to sufficiently cope with medical and surgical stressors. Even without an intervention, a frail individual is at risk for falls, disability, comorbid disease, delirium, cognitive decline, iatrogenic complications, social withdrawal, and death. Most of the common surgical procedures including hip, back, and cardiac surgeries are all associated with transitions in frailty [1]. Few articles have addressed the impact of perioperative frailty in the elderly, even though majority of the Americans undergo surgeries in the year before their death, when the physiological reserves are on a decline.

Making a surgical decision in frail patients is complex. The balance between high operative risk and the potential benefits of surgery is not always clear. The challenge lies in effectively identifying patients that will benefit most from operative intervention and then optimize them for surgery. In this review, we aim to look at the recent literature addressing surgical outcomes in the frail individuals. We will discuss the pathophysiology of frailty and methods of identifying and risk stratifying the frail individual undergoing a surgical procedure. Future research ideas will also be discussed.

\section{Frailty and Aging}

One of the common definitions of frailty is "the existence of an underlying pathophysiological mechanism responsible for 
the phenotypical manifestation of aging" [2•]. Frailty has commonly been associated with aging, but it is important to differentiate the two. Frailty may or may not be age related and manifests as a decrease in physiological reserve with a poor capacity to maintain homeostasis [3]. The two common models of frailty are the deficit and accumulation/excess models (Fig. 1).

The pathophysiology of frailty is complex and is an amalgamation of multiple cellular responses. Each of these cellular responses plays a role in normal aging and possibly in frailty. The loss of homeostasis increases vulnerability to stressors, and the body attempts to respond by apoptosis, senescence, and repair. A balance or an imbalance between apoptosis and senescence affects multiple systems and leads to healthy aging or frailty. Apoptosis is vital to various phases of embryogenesis. It is also important in the adult life and helps in tissue remodeling by maintaining organ function and size. Insufficient ATP levels lead to a shift from apoptotic to necrotic cell death. A decline in cellular ATP is found to promote age-related cell death over apoptosis, in response to oxidative stress [4]. Centenarians who have aged successfully have cells that are more prone to apoptosis [5].

"Inflamm-aging" as a concept of frailty was described by Franceschi [6]. The degree of inflammatory load appears to parallel the extent of frailty. Pro-inflammatory markers like IL-6, TNF-alpha, and CRP have all been measured in frailty. Frailty status has a stronger association with inflammation than does aging $[7 \bullet \bullet]$. Frailty and aging are accompanied by dysregulation of the ubiquitin-proteasomal system, autophagy, and the crosstalk between both systems [8]. A relatively new focus of study in aging and frailty is monocyte/macrophage-mediated immune activations. Neopterin, a marker of immune activation independent of IL-6, correlates with frailty. Another relatively new finding is that vitamin $\mathrm{D}$ deficiency $<15 \mathrm{ng} \mathrm{mL}$ increases the odds of frailty by four times $\left[8,9,10^{\bullet}, 11\right]$. Homeostatic responses affect aging and frailty via the abovementioned processes.

\section{Measures of Frailty}

It is common for clinicians to think of frailty in a "I know it when I see it" sort of way. Currently in most surgical fields, clinical intuition leads to decisions regarding considering a particular patient a surgical candidate. These assessments, while unsupported by objective measures, are frequently accurate. Regardless, there are now several tools and evaluation

Fig. 1 Models of frailty

\section{Deficit Driven Model}

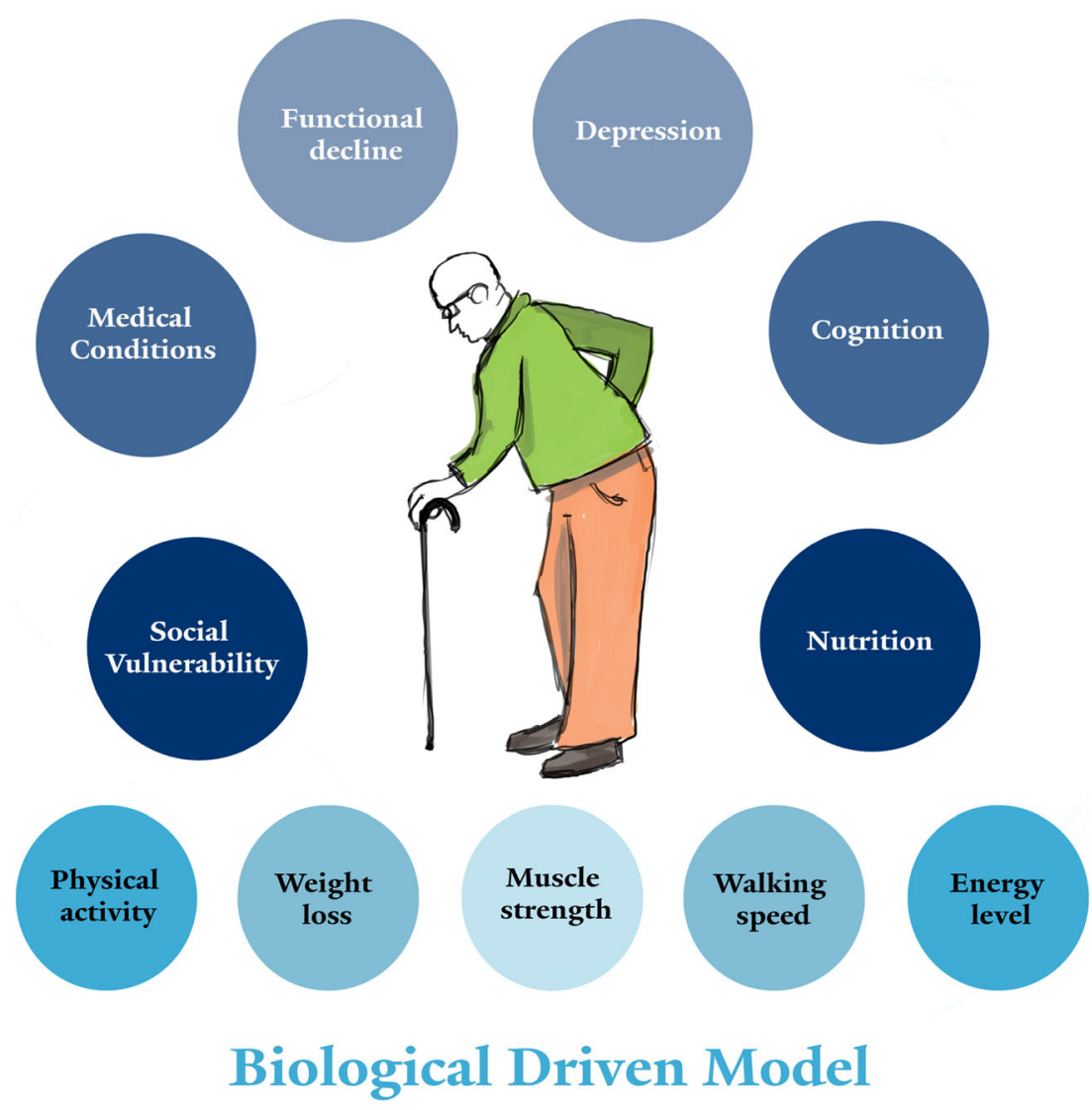


techniques to utilize in evaluating a potentially frail surgical candidate.

The gold standard for frailty in older patients is a comprehensive geriatric assessment (CGA), which involves a multidisciplinary, holistic approach. It is not a singular test, or a battery of assessments. Rather, it is approached as a process of care that requires multiple hours of assessment. As such, most practices will have neither the time nor the professional resources to perform a CGA on most, if any, of their patients. Therefore, there is a desire and need for a simplified frailty assessment to utilize as a screening tool in the clinics. Many of the CGA elements are evaluated by specialists with advanced training and experience. For frailty assessments in preoperative evaluation to be routine, tests and instruments that are simplified and can easily be conducted and interpreted by anesthesiologists are needed.

While simplified frailty tools facilitate assessments in the clinic, these metrics sacrifice accuracy. At a minimum, these simplified assessment tools can be additive and supportive to the standard preanesthetic or presurgical evaluation. Numerous tools have been developed to identify frailty. These tools vary significantly in regard to the level of training required of the test examiner and the amount of time necessary to complete the assessment. A review of the more commonly used metrics follows.

Several assessments can be completed completely without examining the patient as part of the evaluation - often questionnaires; these can be easily administered via telephone interviews.

\section{Karnofsky Performance Status Scale}

The Karnofsky performance status scale (KPS) looks at functional impairment in 10\% increments, from 100\% (normal, no complaints, no evidence of disease) to $0 \%$ (dead), as well as three corresponding levels of limitation. The three-level version of the scale is supposed to have a higher inter-rater reliability score. The scoring is as follows:

- Normal/no limitations (minimal or no symptoms of disease $[\geq 80 \%$ function])

- Mild/moderate (variable assistance in daily activities [5070\%])

- Severe functional impairment/disability (disabled, hospitalization indicated, and/or moribund $[\leq 40 \%]$ )

\section{Risk Analysis Index}

Risk Analysis Index is another tool that has been used for measuring frailty in surgical populations. This score is an adaptation of the Minimum Data Set (MDS) Mortality Risk Index-Revised (MMRI-R). RAI-C is a novel way of measuring frailty prospectively using a questionnaire. RAI$\mathrm{A}$ is a retrospective way of measuring frailty using variables from the Veterans Affairs or American College of Surgeons National Surgical Quality Improvement Projects. RAI-C has proved feasible for system-wide screening with good predictive power. A subsample has shown similar predictive power between the RAI-C, RAI-A, and modified Frailty Index $[12 \bullet \bullet$.

\section{The Fried Frailty Assessment}

The Frailty Assessment designed by Fried and colleagues has emerged as the gold standard for relatively rapid assessment of the frailty phenotype. This clinical test is a simple, straightforward, assessment covering five aspects of frailty, namely weight loss, exhaustion, low physical activity, slowness, and weakness. Any clinician can complete the Fried Frailty Assessment in under $10 \mathrm{~min}$ and with a minimum investment in equipment. Patients are scored between 0 and 5 , with each of the metrics counting equally to the composite result. A score $\geq 3$ is indicative of frailty.

\section{Modified Frailty Index}

This scoring system was first described by Saxton and Velanovich [13] Score is based on 11 variables present in the Canadian Study of Health and Aging Frailty Index (CSHAFI). Modified frailty index (mFI) is a simple tool that has shown to be predictive of postoperative complication. This score can be easily obtained from patient's history and physical exam.

\section{Frailty and Perioperative Surgical Outcomes}

The American Society of Anesthesiologist-physical status (ASA-PS) classification is routinely used as one of the predictors of operative risk and has been used as such for nearly 80 years. Frailty is an independent risk factor for in-hospital mortality and morbidity. A multidimensional frailty score has shown to be a better predictor of outcomes in geriatric patients who undergo surgery [14]. Frailty scores have recently been incorporated in perioperative medicine to predict postoperative recovery and outcomes.

To discuss postoperative outcomes, one can divide the recovery phase of surgery into three distinctive phases $[15 \bullet \cdot]$.

- Early phase (first $24 \mathrm{~h}$ to 7 days) - the early phase is influenced mostly by pain, nausea, perioperative medications, and delirium.

- Intermediate phase (28 days to 60 days) - this phase is influenced mostly by pain, anxiety and depression, physical impairment, and cognitive dysfunction. 
- Late postoperative phase (6 weeks to 3 months) _ problem suffered in the early and intermediate phase can persist into this phase.

Frail patients are at risk during each and every phase of recovery. These patients might survive their operations and intensive care unit (ICU) stay but have an increased mortality of $48 \mathrm{vs}$. $25 \%$ for non-frail patients at 12 months. Only $15 \%$ of the frail patients continued to live independently after surgery [16]. The typical recovery trajectory is characterized by an initial abrupt decline in function, followed by progressive resolution towards the original state or a new equilibrium. Preoperative risk stratification can identify patients' risks of postoperative death, functional decline, and reduced life expectancy.

Multiple investigators have looked at the effects of frailty in various surgical subspecialties. Isharwal et al. applied the Risk Analysis Index (RAI) to the National Surgical Quality Improvement Program (NSQIP) in order to determine the effect of preoperative frailty in urologic surgical patients. RAI scores $>35$ were associated with a mortality of $29.51 \%$; when compared to patients with a RAI score $<6$, the mortality was $0.47 \%$. This group also found increased complications, length of stay, and 30-day readmission rate and decreased discharge to home in the patient with higher RAI scores. Discharge to home was 33\% in RAI scores $>35$ and $94 \%$ in RAI scores $<6$. Patients were discharged to skilled nursing, acute care, and rehabilitation facilities [17]. Dolgin et al. used the KPS to represent frailty at the time of transplant. They studied 24,505 liver transplant recipients and found that severely impaired patients had a 30-day mortality of nearly double than that of patients with moderate impairment (5.2 vs. 2. $2 \%$ ). Proportions of patients that died on day 0 or 1 were more than four times than those with moderate impairment [18].

A common population that we deal with frequently is the frail geriatric patient presenting for or has had an orthopedic spine and hip surgical procedure. Leven et al. looked at $\mathrm{mFI}$ and applied it to the NSQIP database. They found increased mortality, blood transfusion, pulmonary embolus, and deep vein thrombosis rates in patients with a higher mFI. As the $\mathrm{mFI}$ scores increased from 0 to 0.27 , mortality increased from 0.3 to $10 \%$. Blood transfusion risk increased from 32 to $55 \%$.

Shin et al. found an increased incidence of Clavien-Dindo grade IV complications (cardiac arrest, myocardial infarction, septic shock, pulmonary embolism, postoperative dialysis, reintubation, and prolonged ventilator requirement) with a $\mathrm{mFI}>0.45$ [19]. Cognitive impairment has been associated with frailty and poor outcomes. In one study, frail individuals with a low Minicog assessment had a 68\% incidence of delirium [20]. Frailty has been assessed in patients undergoing cardiac surgery. There seems to be a twofold increased risk of delirium in frail individuals after cardiac surgery [21]. Kotajarvi and his colleagues looked at patients following aortic valve replacement. They found a greater percentage of frail (37\%) compared to non-frail $(18 \%)$ participants were rehospitalized one or more times. One-year all-cause mortality following hospital discharge trended to be higher in frail (13\%), compared to non-frail $(4.3 \%)$ patients. A surprising finding was that the frail participants exhibited significantly improved Duke Activity Status Index (50\% improvement) and Physical Component Scale (14\% improvement) scores at 3 months postdischarge. Non-frail participants had significantly higher mental health by Mental Component Scale than frail peers at baseline (54.9 vs. 49.4). Three months after surgery, scores for the non-frail group improved less than a point. On the other hand, scores for the frail group trended towards improvement. At baseline, non-frail participants had significantly higher physical well-being, social activity, and quality of life, than their frail peers. When combined, study participants demonstrated significant improvements in all measures of wellbeing, social activity, and overall quality of life after surgery [22]. Further analysis revealed that frail participants improved to a greater extent than non-frail participants in physical well-being and quality of life. As a result, no differences were observed between non-frail and frail individuals in any measure of wellbeing or quality of life at follow-up [22]. Quality of life prior to surgery bordered the threshold of 50 among frail participants, a score below which interventions are warranted [22]. However, this important measure increased an impressive 22 points following surgery, a change that far exceeds a clinically important difference. These data suggest that compromised physical health, well-being, and quality of life can be markedly and meaningfully improved by surgery in higher risk older adults [22].

\section{Infections in Frail Surgical Patients}

Frailty is a multisystem disorder leading to maladaptive response to stressors [23]. Frail individuals are at increased risk for postoperative infections. Poor wound healing as a result of poor nutrition can be complicated by surgical site infection. Cognitive impairment increases risk for aspiration pneumonia, while poor mobility can result in pressure ulcers. A high mFI score at time of surgery was noted to have increased risk for pneumonia, surgical site infections, and urinary tract infections $[19,24,25,26 \bullet, 27]$. Sarcopenia, which is a marker of frailty, has been associated with nosocomial infection in the elderly [28]. In select surgical patients, such as patients who undergo colorectal cancer resection, hepatic resection, and liver transplantation, investigators were able to demonstrate the association of sarcopenia with postsurgical infections [29-31].

\section{Presurgical Intervention to Improve Outcomes of Frail Adults}

The functional decline can occur after a stressful surgical intervention especially in individuals who are already 
considered frail. Interventions should be targeted to a particular aspect of recovery that has led to functional decline. To target this particular aspect of failure (e.g., poor nutritional intake), one must use domain-specific dichotomous measures of recovery, rather than a composite score. Failure in one domain is often accompanied by failure in another domain. For instance, a patient may fail to recover cognitively because they have been in pain and subsequently received opioid analgesia $[15 \bullet \bullet]$.

There is an increasing body of literature that demonstrates the role of physical therapy or exercise, to improve baseline functional reserve of the patient who will undergo surgery. Exercise training can improve the skeletal adaptation by increasing mitochondrial content and respiratory capacity of muscle fibers, improve blood perfusion to working tissue by increasing cardiac output and systolic blood pressure, and improve blood oxygenation by increasing breathing rate and depth $[32,33]$. Increasing the physical activity status can improve postoperative outcomes (length of stay, infection rates, etc.) of selected surgical patients. Preoperative physical therapy applied to cardiac surgery patients has reduced the risk for postoperative atelectasis and pneumonia [34]. Preoperative pulmonary rehabilitation improved exercise capacity, dyspnea, and health-related quality of life of patients requiring lung volume reduction surgery and lung transplantation [35].

A meta-analysis of patients who underwent hip replacement demonstrated reduced pain and improved physical function with preoperative exercise intervention [36]. Prehabilitation consisting of aerobic exercise, resistance training, and inspiratory muscle training among individuals before intraabdominal surgery has been associated with decreased pulmonary complications after surgery [37••]. Prehabilitation that addressed nutritional status by counseling and protein supplementation in addition to exercise has shown improvement in postoperative functional recovery of patients undergoing colorectal cancer surgery [38]. In a systematic review, health-related quality of life improvement and reduction in fatigue were noted among cancer patients who underwent preoperative exercise training [39॰]. Patients who underwent lung cancer resection and received preoperative pulmonary rehabilitation of patients have improved preoperative functional capacity, decreased postoperative respiratory morbidity, shorter length of stay, and shorter duration of chest tube use [40].

Poor nutritional status has been associated with frailty [41]. Patients undergoing surgery with malnutrition have increased the risk for postoperative complication, prolonged hospital stay, higher readmission rate, delayed bowel function recovery, and increased postoperative mortality [42]. Implementation of nutrition screening protocol in the preoperative evaluation is important. Presurgical nutrition therapy can optimize an individual's nutritional reserve adequate to compensate for the catabolic response to surgery [43•]. There is insufficient evidence to recommend a specific nutritional intervention at present (i.e., enteral nutrition, parenteral nutrition, or oral supplement) for at-risk patients [44].

\section{Recommendations for Future}

A meta-analysis should be undertaken to study the impact of frailty in a surgical candidate. One needs to understand that some frail patients will improve after surgery while others will decline. Further prospective trials need to be conducted to help us guide therapy. Each frailty trajectory and outcome should be studied, and then, prehabilitation should focus on improving those outcomes. There needs to be a focus on the development of a surgical frailty score that would break up frailty into modifiable and non-modifiable factors. Research is lacking on the effects of intraoperative variables on frailty. Intraoperative drug dosing and hemodynamic monitoring should be optimized for the frail individual. Psychosocial factors play an import role in outcomes after surgery. The link between psychosocial factors and frailty could explain why patients with similar frailty scoring fare differently after similar procedures. Studies focusing on vitamin D, apoptosis, senescence, and repair seen in healthy aging could give us valuable insight into the pathophysiology of frailty. Finally incorporating frailty scoring in routine preoperative assessment would significantly enhance our ability to fill gaps in our knowledge about frailty, frailty trajectories after surgery, and postoperative outcomes.

\section{Conclusion}

Frailty score is associated with predicting postoperative outcomes including frailty trajectories. Prior to a procedure, one must not only use the usual risk stratification strategies but should also consider using frailty scores. These scores may help patients and their families make realistic informed decisions about the effect of surgery and its possible effect to their quality of life. If the patient decides on the surgery, then practitioners may need to utilize additional resources like a geriatric team, intensive care unit, and alternative levels of care. Understanding of frailty and its trajectories will lead to earlier palliative care discussion, implementation of care team-based pathways, and tailored anesthesia approach.

\section{Compliance with Ethical Standards}

Conflict of Interest Ranjit Deshpande, William Stuart Asch, and Maricar Malinis declare they have no conflict of interest.

Human and Animal Rights and Informed Consent This article does not contain any studies with human or animal subjects performed by any of the authors. 


\section{References}

Papers of particular interest, published recently, have been highlighted as:

- Of importance

•- Of major importance

1. Bandeen-Roche K, Seplaki CL, Huang J, Buta B, Kalyani RR, Varadhan R, et al. Frailty in older adults: a nationally representative profile in the United States. J Gerontol A Biol Sci Med Sci. 2015;70(11):1427-34.

2. Ferrucci L, Fabbri E, Walston JD. Frailty. In: Halter JB, Ouslander JG, Studenski S, High KP, Asthana S, Supiano MA, et al., editors. Hazzard's geriatric medicine and gerontology. 7th ed. New York: McGraw-Hill Education; 2017. Good chapter to review the basics of frailty.

3. Fried LP, Ferrucci L, Darer J, Williamson JD, Anderson G. Untangling the concepts of disability, frailty, and comorbidity: implications for improved targeting and care. J Gerontol A Biol Sci Med Sci. 2004;59(3):255-63.

4. Miyoshi N, Oubrahim H, Chock PB, Stadtman ER. Age-dependent cell death and the role of ATP in hydrogen peroxide-induced apoptosis and necrosis. Proc Natl Acad Sci U S A. 2006;103(6):172731.

5. Franceschi C, Monti D, Scarfi MR, Zeni O, Temperani P, Emilia G, et al. Genomic instability and aging. Studies in centenarians (successful aging) and in patients with Down's syndrome (accelerated aging). Ann N Y Acad Sci. 1992;663:4-16.

6. Franceschi C, Campisi J. Chronic inflammation (inflammaging) and its potential contribution to age-associated diseases. J Gerontol A Biol Sci Med Sci. 2014;69(Suppl 1):S4-9.

7.• Van Epps P, Oswald D, Higgins PA, Hornick TR, Aung H, Banks RE, et al. Frailty has a stronger association with inflammation than age in older veterans. Immun Ageing. 2016;13:27. This paper suggests that frailty status has a stronger association with inflammation and inflammatory markers than age.

8. Hohn A, Weber D, Jung T, Ott C, Hugo M, Kochlik B, et al. Happily (n)ever after: aging in the context of oxidative stress, proteostasis loss and cellular senescence. Redox Biol. 2017;11: 482-501.

9. Krams T, Cesari M, Guyonnet S, Abellan Van Kan G, Cantet C, Vellas $\mathrm{B}$, et al. Is the 25-hydroxy-vitamin $\mathrm{D}$ serum concentration a good marker of frailty? J Nutr Health Aging. 2016;20(10):1034-9.

10. Buta B, Choudhury PP, Xue QL, Chaves P, Bandeen-Roche K, Shardell M, et al. The association of vitamin D deficiency and incident frailty in older women: the role of cardiometabolic diseases. J Am Geriatr Soc. 2017;65(3):619-24. When one accounts for the cardiometabolic disorder in frail women there seems to be no association of serum vitamin levels and frailty.

11. Bruyere O, Cavalier E, Buckinx F, Reginster JY. Relevance of vitamin D in the pathogenesis and therapy of frailty. Curr Opin Clin Nutr Metab Care. 2017;20(1):26-9.

12.• Hall DE, Arya S, Schmid KK, Blaser C, Carlson MA, Bailey TL, et al. Development and initial validation of the risk analysis index for measuring frailty in surgical populations. JAMA Surg. 2017;152(2):175-82. Risk Analysis index can be as a clinical questionnaire, or retrospectively using variables from the surgical quality improvement databases to measure frailty.

13. Saxton A, Velanovich V. Preoperative frailty and quality of life as predictors of postoperative complications. Ann Surg. 2011;253(6): 1223-9.

14. Kim SW, Han HS, Jung HW, Kim KI, Hwang DW, Kang SB, et al. Multidimensional frailty score for the prediction of postoperative mortality risk. JAMA Surg. 2014;149(7):633-40.
15.• Bowyer AJ, Royse CF. Postoperative recovery and outcomeswhat are we measuring and for whom? Anaesthesia. 2016;71(Suppl 1):72-7. This is a good review to understand how to measure recovery and the currently available tools.

16. Wang W, Bagshaw SM, Norris CM, Zibdawi R, Zibdawi M, MacArthur R, et al. Association between older age and outcome after cardiac surgery: a population-based cohort study. J Cardiothorac Surg. 2014;9:177.

17. Isharwal S, Johanning JM, Dwyer JG, Schimid KK, LaGrange CA. Preoperative frailty predicts postoperative complications and mortality in urology patients. World J Urol. 2017;35(1):21-6.

18. Dolgin NH, Martins PN, Movahedi B, Lapane KL, Anderson FA, Bozorgzadeh A. Functional status predicts postoperative mortality after liver transplantation. Clin Transpl. 2016;30(11):1403-10.

19. Shin JI, Keswani A, Lovy AJ, Moucha CS. Simplified frailty index as a predictor of adverse outcomes in total hip and knee arthroplasty. J Arthroplast. 2016;31(11):2389-94.

20. Kistler EA, Nicholas JA, Kates SL, Friedman SM. Frailty and short-term outcomes in patients with hip fracture. Geriatr Orthop Surg Rehabil. 2015;6(3):209-14.

21. Brown CH, Max L, LaFlam A, Kirk L, Gross A, Arora R, et al. The association between preoperative frailty and postoperative delirium after cardiac surgery. Anesth Analg. 2016;123(2):430-5.

22. Kotajarvi BR, Schafer MJ, Atkinson EJ, Traynor MM, Bruce CJ, Greason KL, et al. The impact of frailty on patient-centered outcomes following aortic valve replacement. J Gerontol A Biol Sci Med Sci. 2017;72(7):917-21.

23. Chen X, Mao G, Leng SX. Frailty syndrome: an overview. Clin Interv Aging. 2014;9:433-41.

24. Karam J, Tsiouris A, Shepard A, Velanovich V, Rubinfeld I. Simplified frailty index to predict adverse outcomes and mortality in vascular surgery patients. Ann Vasc Surg. 2013;27(7):904-8.

25. Tsiouris A, Hammoud ZT, Velanovich V, Hodari A, Borgi J, Rubinfeld I. A modified frailty index to assess morbidity and mortality after lobectomy. J Surg Res. 2013;183(1):40-6.

26. Ali R, Schwalb JM, Nerenz DR, Antoine HJ, Rubinfeld I. Use of the modified frailty index to predict 30-day morbidity and mortality from spine surgery. J Neurosurg Spine. 2016;25(4):537-41. This study shows us the utility of modified frailty index in spine procedures.

27. Partridge JS, Fuller M, Harari D, Taylor PR, Martin FC, Dhesi JK. Frailty and poor functional status are common in arterial vascular surgical patients and affect postoperative outcomes. Int J Surg. 2015;18:57-63.

28. Cosqueric G, Sebag A, Ducolombier C, Thomas C, Piette F, WeillEngerer S. Sarcopenia is predictive of nosocomial infection in care of the elderly. Br J Nutr. 2006;96(5):895-901.

29. Lieffers JR, Bathe OF, Fassbender K, Winget M, Baracos VE. Sarcopenia is associated with postoperative infection and delayed recovery from colorectal cancer resection surgery. Br J Cancer. 2012;107(6):931-6.

30. Krell RW, Kaul DR, Martin AR, Englesbe MJ, Sonnenday CJ, Cai $\mathrm{S}$, et al. Association between sarcopenia and the risk of serious infection among adults undergoing liver transplantation. Liver Transpl. 2013;19(12):1396-402.

31. Valero V 3rd, Amini N, Spolverato G, Weiss MJ, Hirose K, Dagher $\mathrm{NN}$, et al. Sarcopenia adversely impacts postoperative complications following resection or transplantation in patients with primary liver tumors. J Gastrointest Surg. 2015;19(2):272-81.

32. Holloszy JO, Coyle EF. Adaptations of skeletal muscle to endurance exercise and their metabolic consequences. J Appl Physiol Respir Environ Exerc Physiol. 1984;56(4):831-8.

33. Carli F, Scheede-Bergdahl C. Prehabilitation to enhance perioperative care. Anesthesiol Clin. 2015;33(1):17-33. 
34. Hulzebos EH, Smit Y, Helders PP, van Meeteren NL. Preoperative physical therapy for elective cardiac surgery patients. Cochrane Database Syst Rev. 2012;11:CD010118.

35. Takaoka ST, Weinacker AB. The value of preoperative pulmonary rehabilitation. Thorac Surg Clin. 2005;15(2):203-11.

36. Gill SD, McBurney H. Does exercise reduce pain and improve physical function before hip or knee replacement surgery? A systematic review and meta-analysis of randomized controlled trials. Arch Phys Med Rehabil. 2013;94(1):164-76.

37.• Moran J, Guinan E, McCormick P, Larkin J, Mockler D, Hussey J, et al. The ability of prehabilitation to influence postoperative outcome after intra-abdominal operation: a systematic review and meta-analysis. Surgery. 2016;160(5):1189-201. This study shows prehabilitation appears to decrease the incidence of postoperative complications.

38. Li C, Carli F, Lee L, Charlebois P, Stein B, Liberman AS, et al. Impact of a trimodal prehabilitation program on functional recovery after colorectal cancer surgery: a pilot study. Surg Endosc. 2013;27(4):1072-82.

39. Loughney L, Grocott MP. Exercise and nutrition prehabilitation for the evaluation of risk and therapeutic potential in cancer patients: a review. Int Anesthesiol Clin. 2016;54(4):e47-61. This study suggests that prehabilitation programs should be initiated at cancer diagnosis and be based on 3 components, including physical fitness/activity interventions, nutritional support, and psychological support up to the point of surgery.

40. Morano MT, Araujo AS, Nascimento FB, da Silva GF, Mesquita R, Pinto JS, et al. Preoperative pulmonary rehabilitation versus chest physical therapy in patients undergoing lung cancer resection: a pilot randomized controlled trial. Arch Phys Med Rehabil. 2013;94(1):53-8.

41. Artaza-Artabe I, Saez-Lopez P, Sanchez-Hernandez N, FernandezGutierrez N, Malafarina V. The relationship between nutrition and frailty: effects of protein intake, nutritional supplementation, vitamin D and exercise on muscle metabolism in the elderly. A systematic review. Maturitas. 2016;93:89-99.

42. West MA, Wischmeyer PE, Grocott MPW. Prehabilitation and nutritional support to improve perioperative outcomes. Curr Anesthesiol Rep. 2017;7(4):340-9.

43. Gupta R, Gan TJ. Preoperative nutrition and prehabilitation. Anesthesiol Clin. 2016;34(1):143-53. A good review looking at prehabilitation in specific population.

44. Burden S, Todd C, Hill J, Lal S. Pre-operative nutrition support in patients undergoing gastrointestinal surgery. Cochrane Database Syst Rev. 2012;11:CD008879. 